\title{
Random Walks and Effective Resistances on Toroidal and Cylindrical Grids
}

\author{
Monwhea Jeng \\ momo@physics.ucsb.edu \\ Physics Department \\ University of California, Santa Barbara, CA 93106-4030
}

September 1, 2021

\begin{abstract}
A mapping between random walk problems and resistor network problems is described and used to calculate the effective resistance between any two nodes on an infinite twodimensional square lattice of unit resistors. The superposition principle is then used to find effective resistances on toroidal and cylindrical square lattices.
\end{abstract}

\section{Introduction}

There is an interesting but little-known correspondence between properties in random walk problems and properties in electric network problems [1. In this paper we describe this correspondence and show how it can be used to calculate resistances between arbitrary nodes on an infinite two-dimensional square lattice of unit resistors. While this problem has been solved elsewhere [2]- 10], the treatment here both shows the value of mapping electric network 
problems to random walk problems, and puts the answer in a form that can, by use of the superposition principle, be used to calculate resistances on toroidal and cylindrical grids.

\section{Random Walks and Effective Resistances}

In this section we demonstrate a number of surprising relationships between resistor networks and certain random walk problems. A very lucid explanation of the results covered here, as well as other aspects of this mapping, can be found in [1].

We first consider a general finite connected resistor network (Fig. 1). If $x$ and $y$ are connected nodes, let the resistor connecting them have resistance $r_{x y}$. We now consider a random walker who goes from site to site, weighing each possible step by its inverse resistance. To be specific, if $N(x)$ is the set of all nodes connected to $x$ by a single resistor, then the probability that a random walker at $x$ will next move to the node $y \in N(x)$ is

$$
p_{x \rightarrow y}=\frac{1}{c_{x} r_{x y}}, \quad \text { where } c_{x} \equiv \sum_{y \in N(x)} \frac{1}{r_{x y}}
$$

Now put nodes $A$ and $B$ at voltages 1 and 0 , and let current flow through the network, with no sources at nodes besides $A$ and $B$. Then $V_{x}$, the voltage at an arbitrary point $x$, can be interpreted as the probability that the above random walker, starting at $x$, will get to $A$ before $B$. To see this, we first note that this probability interpretation clearly works at the boundary conditions $V_{A}=1$ and $V_{B}=0$. At other points, $y \neq A$ or $B$, there is no current source, so from Kirchoff's laws,

$$
\begin{aligned}
0 & =\sum_{y \in N(x)} I_{x \rightarrow y}=\sum_{y \in N(x)} \frac{V_{x}-V_{y}}{r_{x y}}=V_{x} \sum_{y \in N(x)} \frac{1}{r_{x y}}-\sum_{y \in N(x)} \frac{V_{y}}{r_{x y}}= \\
& =c_{x}\left(V_{x}-\sum_{y \in N(x)} p_{x \rightarrow y} V_{y}\right)
\end{aligned}
$$


And $V_{x}=\sum_{y \in N(x)} p_{x \rightarrow y} V_{y}$ is exactly the relationship that we would write down for the probability $V_{x}$ that a random walker starting at $x$ would reach $A$ before $B$. Since both the resistor and random walk problems have the same boundary conditions and solve the same linear equations, they have the same unique solution (although, technically, for an infinite lattice the solution is not unique - see section 3 for more details).

We now calculate the current from $A$ to $B$ :

$$
\begin{aligned}
I & =\sum_{y \in N(A)} I_{A \rightarrow y}=\sum_{y \in N(A)} \frac{V_{A y}}{r_{A y}}=c_{A} \sum_{y \in N(A)} p_{A \rightarrow y}\left(1-V_{y}\right)= \\
& =c_{A} \sum_{y \in N(A)} p_{A \rightarrow y} \times(\text { probability that a random walker at } y \text { gets to } B \text { before } A) \\
& =c_{A} p_{A B}
\end{aligned}
$$

where we have used the random walk mapping, and defined $p_{A B}$ as the probability that a random walker, starting at $A$, gets to $B$ before returning to $A$.

The voltage between $A$ and $B$ is 1 , and the current is given by equation 3 so from Ohm's law, the effective resistance between $A$ and $B$ is

$$
R_{A B}=\frac{1}{c_{A} p_{A B}}
$$

It will be useful to write this result in a different form. For a random walker starting at A, let $\Delta_{A B}$ be the expectation value of the number of vists to $\mathrm{A}$ minus the number of vists to $\mathrm{B}$, after infinitely many steps. If $P_{n}(x)$ is the probability that after $\mathrm{n}$ steps the walker will be at node $\mathrm{x}$, then

$$
\Delta_{A B}=\sum_{n=0}^{\infty}\left(P_{n}(A)-P_{n}(B)\right)
$$

It is not hard to show from the definition of $\Delta_{A B}$, that $\Delta_{A B}=1 / 2 p_{A B}$, and thus 


$$
R_{A B}=\frac{2}{c_{A}} \Delta_{A B}=\frac{2}{c_{A}} \sum_{n=0}^{\infty}\left(P_{n}(A)-P_{n}(B)\right)
$$

\section{$3 \quad R_{e f f}$ on an Infinite Grid}

In this section we show how the random walk mapping can be used to find effective resistances on an infinite two-dimensional grid of unit resistors. This problem has been solved elsewhere [2] [10], but we rederive the result here to demonstrate the power of the mapping described above.

We can solve the corresponding random walk problem with a generating function [13]. Let the random walker start at position $(0,0)$. After $N$ timesteps she is at position $\vec{x}_{N}=\sum_{i=1}^{N} \hat{e}_{i}$, where $\hat{e}_{i}$ is the step at timestep $i$, and each $\hat{e}_{i}$ is chosen with equal probability from $(0,1)$, $(0,-1),(1,0)$, and $(-1,0)$. Then the expectation value of $e^{i \hat{e} \cdot \vec{\theta}}$, where $\hat{e}$ is any step, and $\vec{\theta}$ is a 2 -vector, is

$$
\begin{gathered}
\phi(\vec{\theta}) \equiv E\left(e^{i \hat{e} \cdot \vec{\theta}}\right)=\frac{1}{2}\left(\cos \theta_{x}+\cos \theta_{y}\right), \text { while } \\
E\left(e^{i \vec{x}_{N} \cdot \vec{\theta}}\right)=E\left(\prod_{i=1}^{N} e^{i \hat{e}_{i} \cdot \vec{\theta}}\right)=[\phi(\vec{\theta})]^{N}=\phi^{N}(\theta)
\end{gathered}
$$

Fourier tranforming, the probability of being at $\vec{x}$ at timestep $N$ is

$$
P_{N}(\vec{x})=E\left(\delta_{\vec{x}, \vec{x}_{N}}\right)=\frac{1}{(2 \pi)^{2}} \int_{-\pi}^{\pi} d \theta_{x} \int_{-\pi}^{\pi} d \theta_{y} e^{-i \vec{x} \cdot \vec{\theta}} \phi^{N}(\vec{\theta})
$$

Let $\Delta_{m n}^{\infty \infty} \equiv \Delta_{(0,0),(m, n)}$. The " $\infty \infty$ " superscript indicates that the grid is infinite in both length and width, and $\Delta_{(0,0),(m, n)}$ was defined in the last section.

$$
\Delta_{m n}^{\infty \infty}=\sum_{N=0}^{\infty}\left(P_{N}(0,0)-P_{N}(m, n)\right)
$$




$$
\begin{aligned}
& =\frac{1}{(2 \pi)^{2}} \int_{-\pi}^{\pi} d \theta_{x} \int_{-\pi}^{\pi} d \theta_{y}\left(1-e^{-i(m, n) \cdot \vec{\theta}}\right) \sum_{N=0}^{\infty} \phi^{N}(\vec{\theta}) \\
& =\frac{1}{(2 \pi)^{2}} \int_{0}^{2 \pi} d \theta_{x} \int_{0}^{2 \pi} d \theta_{y} \frac{1-e^{-i(m, n) \cdot \vec{\theta}}}{1-\phi(\vec{\theta})} \\
R_{m n}^{\infty \infty} & =\frac{1}{8 \pi^{2}} \int_{0}^{2 \pi} d x \int_{0}^{2 \pi} d y \frac{1-\cos (m x+n y)}{1-\frac{1}{2}(\cos x+\cos y)}
\end{aligned}
$$

In the last line we have used the mapping in section 2 to turn the random walk quantity $\Delta_{m n}^{\infty \infty}$ into $R_{m n}^{\infty \infty}$, the effective resistance between $(0,0)$ and $(m, n)$. We can get $R_{m n}^{\infty \infty}$ in closed form for any $(m, n)$. We find $R_{01}^{\infty}=\frac{1}{2}$, either by evaluating the integral above, or more simply, by exploiting the symmetry of the original problem [11, 12. For $m=n$ we can evaluate the integral exactly [13, 4, 5], getting $R_{m m}^{\infty \infty}=\frac{2}{\pi} \sum_{i=1}^{m} \frac{1}{2 i-1}$. From these values of $R_{m n}^{\infty \infty}$, we can use the recursion relation $R_{m, n+1}^{\infty \infty}+R_{m, n-1}^{\infty \infty}+R_{m+1, n}^{\infty \infty}+R_{m-1, n}^{\infty \infty}=4 R_{m, n}^{\infty \infty}$ for $(m, n) \neq(0,0)$ (easily derivable from equation 11), to get an exact expression for any $R_{m n}^{\infty \infty}$. As we will see in the next section, the above integral form of $R_{m n}^{\infty \infty}$ is useful for calculating effective resistances on toroidal grids.

If we wish to be rigorous, we should note that for an infinite resistor network, Kirchoff's laws do not have a unique solution. They do however have a unique physical solution, obtainable by requiring that the total power dissipitated be finite and that the current flow be the limit of a sequence of flows contained in finite subnetworks. A rigorous theory of flows in general infinite networks can be found in [14, 15], while analyses specific for the infinite square lattice can be found in $[3,[5]$.

\section{$4 \quad R_{e f f}$ for a Toroidal Grid}

With the solution to the infinite grid, we now turn out attention to the new problem of a toroidal grid of unit resistors. We let the toroidal grid be $M$ by $N$, and want to find $R_{m n}^{M N}$, the effective resistance between nodes $(0,0)$ and $(m, n)$. 
First imagine inserting $\left(1-\frac{1}{M N}\right)$ amps at $(0,0)$, and drawing out $\frac{1}{M N}$ amps at every other node (Fig. 2). Let $S_{m n}^{M N}$ be the voltage between $(0,0)$ and $(m, n)$ in this set-up. The set-up in which $\left(1-\frac{1}{M N}\right)$ amps is drawn out at $(m, n)$, and $\frac{1}{M N}$ amps are inserted at all other nodes will, by symmetry, also have voltage $S_{m n}^{M N}$ between $(0,0)$ and $(m, n)$. Superimposing these two solutions, we find that if we insert 1 amp at $(0,0)$, and take out 1 amp at $(m, n)$, we will have voltage $2 S_{m n}^{M N}$ between $(0,0)$ and $(m, n)$, and thus $R_{m n}^{M N}=2 S_{m n}^{M N}$.

Similar reasoning tells us that for an infinite grid, if we insert $1 \mathrm{amp}$ at $(0,0)$ and let it escape to infinity, then the voltage difference between $(0,0)$ and $(m, n)$ will be $R_{m n}^{\infty \infty} / 2[2]$.

We can now calculate $S_{m n}^{M N}$. Because of the periodicity of the $M \times N$ toroidal grid, the voltage drops on the torus when $\left(1-\frac{1}{M N}\right)$ amps are inserted at $(0,0)$ and $\frac{1}{M N}$ amps are drawn out at all other nodes, are the same as the voltage drops on the infinite grid when $\left(1-\frac{1}{M N}\right)$ amps are inserted at $(a M, b N)$ for all integers $a$ and $b$, and $\frac{1}{M N}$ amps are drawn out at all other nodes. So instead of having the left and right ends (and the top and bottom) wrap around in Fig. 2, we have them repeat. We thus define

$$
I_{a b} \equiv\left\{\begin{array}{cc}
1-\frac{1}{M N} & \text { if } \frac{a}{M} \text { and } \frac{b}{N} \text { are both integers } \\
-\frac{1}{M N} & \text { otherwise }
\end{array}\right.
$$

as the current into site $(a, b)$. Each $I_{a b}$ induces a voltage $I_{a b}\left(R_{a-m, b-n}^{\infty \infty} / 2\right)$ at site $(m, n)$, and a voltage $I_{a b}\left(R_{a b}^{\infty \infty} / 2\right)$ at site $(0,0)$. Superimposing these solutions, we get

$$
R_{m n}^{M N}=2 S_{m n}^{M N}=\sum_{a=-\infty}^{\infty} \sum_{b=-\infty}^{\infty} I_{a b}\left(R_{(a, b),(m, n)}^{\infty \infty}-R_{(a, b),(0,0)}^{\infty \infty}\right)
$$

Equations 12 and 13 contain all the physics. The rest is just mathematical manipulation.

$$
R_{m n}^{M N}=\frac{1}{8 \pi^{2}} \sum_{a=-\infty}^{\infty} \sum_{b=-\infty}^{\infty} I_{a b} \int_{0}^{2 \pi} d x \int_{0}^{2 \pi} d y
$$




$$
\begin{aligned}
\frac{\cos (a x+b y)-\cos ((a-m) x+(b-n) y)}{1-\frac{1}{2}(\cos x+\cos y)} \\
=\frac{1}{8 \pi^{2}} \int_{0}^{2 \pi} d x \int_{0}^{2 \pi} d y \frac{1-\cos (m x+n y)}{1-\frac{1}{2}(\cos x+\cos y)} \\
{\left[\sum_{a=-\infty}^{\infty} \sum_{b=-\infty}^{\infty} I_{a b} \cos (a x) \cos (b y)\right] }
\end{aligned}
$$

We can do the sums over $a$ and $b$ exactly, using the following identity :

$$
\begin{gathered}
\sum_{a=-\infty}^{\infty} \cos (a K x)=\lim _{p \rightarrow \infty} \sum_{a=-p}^{p}\left(e^{i K x}\right)^{a}=\lim _{p \rightarrow \infty} \frac{\sin \left(\left(p+\frac{1}{2}\right) K x\right)}{\sin \left(\frac{1}{2} K x\right)} \\
=\frac{2 \pi}{K} \sum_{u=-\infty}^{\infty} \delta\left(x-\frac{2 \pi}{K} u\right)
\end{gathered}
$$

Here we first did the geometric sum exactly, and then used the representation of the Dirac delta function, $\lim _{p \rightarrow \infty} \frac{\sin (p z)}{z}=\pi \delta(z)$. Using this result, we get

$$
\begin{aligned}
& \sum_{a=-\infty}^{\infty} \sum_{b=-\infty}^{\infty} I_{a b} \cos (a x) \cos (b y) \\
& \quad=\frac{4 \pi^{2}}{M N} \sum_{u=-\infty}^{\infty} \sum_{v=-\infty}^{\infty}\left[\delta\left(x-\frac{2 \pi}{M} u\right) \delta\left(y-\frac{2 \pi}{N} v\right)-\delta(x-2 \pi u) \delta(y-2 \pi v)\right]
\end{aligned}
$$

Inserting this back into equation 14 we can immediately do the integrals over $x$ and $y$, getting

$$
R_{m n}^{M N}=\frac{1}{2 M N} \sum_{u=0}^{M-1} \sum_{v=0}^{N-1}{ }^{\prime} \frac{1-\cos \left(2 \pi\left(m \frac{u}{M}+n \frac{v}{N}\right)\right)}{1-\frac{1}{2}\left(\cos \left(2 \pi \frac{u}{M}\right)+\cos \left(2 \pi \frac{v}{N}\right)\right)}
$$

where the prime on the sum indicates that we omit the term $(u, v)=(0,0)$. We note that this formula immediately implies that $R_{01}^{M N}+R_{10}^{M N}=1-\frac{1}{M N}$.

\section{$5 \quad R_{e f f}$ on a Cylindrical Grid}

We can find the results for an infinite cylindrical grid by taking one the of the toroidal lengths to infinity. One of the sums then becomes a Riemannian representation of an integral. For 
example, if $M \rightarrow \infty$ we get

$$
R_{m n}^{\infty N}=\frac{1}{4 \pi N} \int_{0}^{2 \pi} d x \sum_{v=0}^{N-1} \frac{1-\cos \left(m x+2 \pi n \frac{v}{N}\right)}{1-\frac{1}{2}\left(\cos (x)+\cos \left(2 \pi \frac{v}{N}\right)\right)},
$$

which is "halfway between" equations [1] and 17 The integral over $x$ can be done by contour

integration. For example, for $(m, n)=(0,1)$, we use $\int_{0}^{2 \pi} \frac{d x}{k-\cos (x)}=\frac{2 \pi}{\sqrt{k^{2}-1}}$ to get

$$
R_{01}^{\infty N}=\frac{1}{N} \sum_{v=1}^{N-1} \sqrt{\frac{1-\cos \left(2 \pi \frac{v}{N}\right)}{3-\cos \left(2 \pi \frac{v}{N}\right)}}
$$

Calculating $R_{01}^{\infty 2}=\frac{1}{2 \sqrt{2}}$ is a simple freshman physics problem which can be solved by setting up the right recursion relation. But it is more difficult to show that $R_{01}^{\infty 3}=\frac{2}{\sqrt{21}}$. Also, note that by the comment at the end of section $4 R_{10}^{\infty N}=1-R_{01}^{\infty N}$.

\section{Conclusions}

This mapping may be used on any number of resistor problems or random walk problems. Since a resistor problem and its equivalent random walk problem are essentially the same Dirichlet problem, neither framework is inherently simpler. However, certain manipulations may be more intuitive and physically meaningful in one framework than another. For example, the common freshman physics problem of calculating effective resistances on a cube of $1 \Omega$ resistors is best approached by exploiting the symmetry of the cube to join points of equal voltage. Effective resistances on other Platonic solids may be calculated by the same method, or by cleverly superimposing two easily solvable flows [16. While the same manipulations are possible in the equivalent random walk problem, they are not intuitive, and most physicists would find it easiest to solve a random walk problem on an icosohedron by first mapping it to the equivalent resistor problem. 
On the other hand, for infinite lattices, the direct solution of the resistor network by separation of variables has no obvious physical meaning; but in the random walk framework the generating function is both physically meaningful and natural. The various infinite lattices considered in 10] can be solved by changing the generating function (and some prefactors) in equation [10] (We note that exact values for effective resistances between any two points of triangular or honeycomb lattices can be obtained from recursion relations in [17.)

Perhaps the greatest advantage of mapping infinite resistor lattices to random walks is that many difficult random walk problems have already been solved and their solutions are easily accessible. Suppose we wish to calculate $\lim _{l^{2}+m^{2}+n^{2} \rightarrow \infty} R_{l m n}$, the resistance between the origin and infinity for a three-dimensional cubic lattice. The resulting integrals are extraordinarily difficult to evaluate. However, after using the random walk mapping we get

$$
\begin{aligned}
\lim _{l^{2}+m^{2}+n^{2} \rightarrow \infty} R_{l m n} & =\frac{1}{3} E(\# \text { of vists to } \overrightarrow{0} \text { of a random walk starting at } \overrightarrow{0}) \\
& =\frac{1}{16 \pi^{3} \sqrt{6}} \Gamma\left(\frac{1}{24}\right) \Gamma\left(\frac{5}{24}\right) \Gamma\left(\frac{7}{24}\right) \Gamma\left(\frac{11}{24}\right)=0.50546 \ldots
\end{aligned}
$$

simply by copying results from the random walk literature [18, 19].

This work was supported by a UC Regents Fellowship. I would like to thank the referees and editors for pointing out numerous missed references, and Kerry Kuehn for helpful comments. 


\section{References}

[1] Peter G. Doyle and J. Laurie Snell, Random Walks and Electric Networks (Mathematics Association of America, 1984), chapter 3

[2] Giuluo Venezian, "On the resistance between two points of a grid," Am. J. Phys. 62(11), 1000-1004 (1994)

[3] Harley Flanders, "Infinite Networks: II-Resistance in an Infinite Grid," J. Math. Anal. and Applications, 40, 30-35 (1972)

[4] rec.puzzles archive at http://einstein.et.tudelft.nl/ ${ }^{\sim}$ arlet/puzzles/sol.cgi/physics/resistors

[5] David Cameron, "The Square Grid of Unit Resistors," Math. Scientist, 11, 75-82 (1986)

[6] P. E. Trier, "An Electrical Resistance Network and its Mathematical Undercurrents," Inst. Math. and its Applications, 21, 58-60, (Mar/Apr 1985); P. E. Trier, "Correspondence," Inst. Math. and its Applications, 22, 30-31 (Jan/Feb 1986)

[7] A. H. Zemanian, "A Classical Puzzle: The Driving-Point Resistances of Infinite Grids," IEEE Circuits and Systems Magazine, 7-9 (Mar 1984)

[8] B. van der Pol, "The Finite-Difference Analogy of the Periodic Wave Equation and the Potential Equation," Appendix IV in Probability and Related Topics in Physical Sciences (Interscience Publishers, London, 1959) by M. Kac

[9] L. Lavatelli, "The Resistive Net and Finite-Difference Equations," Am. J. Phys. 40, 1246$1257(1972)$

[10] D. Atkinson and F. J. van Steenwijk, "Infinite Resistive Lattices," Am. J. Phys. 67, 486-492 (1999) 
[11] F. J. Bartis, "Let's Analyze the Resistance Lattice," Am. J. Phys., 35, 354-355 (1967)

[12] R. E. Aitchison, "Resistance Between Adjacent Points of Liebman Mesh," Am. J. Phys., $32(7), 566(1964)$

[13] Frank Spitzer, Principles of Random Walk (Springer-Verlag, NY, 1976), 2nd ed.

[14] Harley Flanders, "Infinite Networks: I - Resistive Networks," IEEE Trans. Circ. Theory, CT-18 (3), 326-331 (1971)

[15] Armen H. Zemanian, "Infinite Electrical Networks," Proc. IEEE, 64(1), 6-17 (1974)

[16] F. J. van Steenwijk, "Equivalent Resistors of Polyhedral Resistive Structures," Am. J. Phys. 66 (1), 90-91 (1998)

[17] T. Horiguchi, "Lattice Green's Functions for the Triangular and Honeycomb Lattics," J. Math. Phys., 13(9) 1411-1419 (1972)

[18] G. N. Watson, "Three Triple Integrals," Quarterly J. Math., 10, 266-276 (1939)

[19] M. L. Glasser and I. J. Zucker, "Extended Watson Integrals for the Cubic Lattices," Proc. Natl. Acad. Sci. USA, 74(5), 1800-1801 (1977); "Lattice Sums" in Theoretical Chemistry: Advances and Perspectives, Volume 5 (Academic Press, New York, 1980), p.67-139 
Figure 1 - A generic resistor network

Figure 2 - In and out currents on an $\mathrm{M}$ by $\mathrm{N}$ toroidal grid of unit resistors, for $\mathrm{M}=4, \mathrm{~N}=3$ 


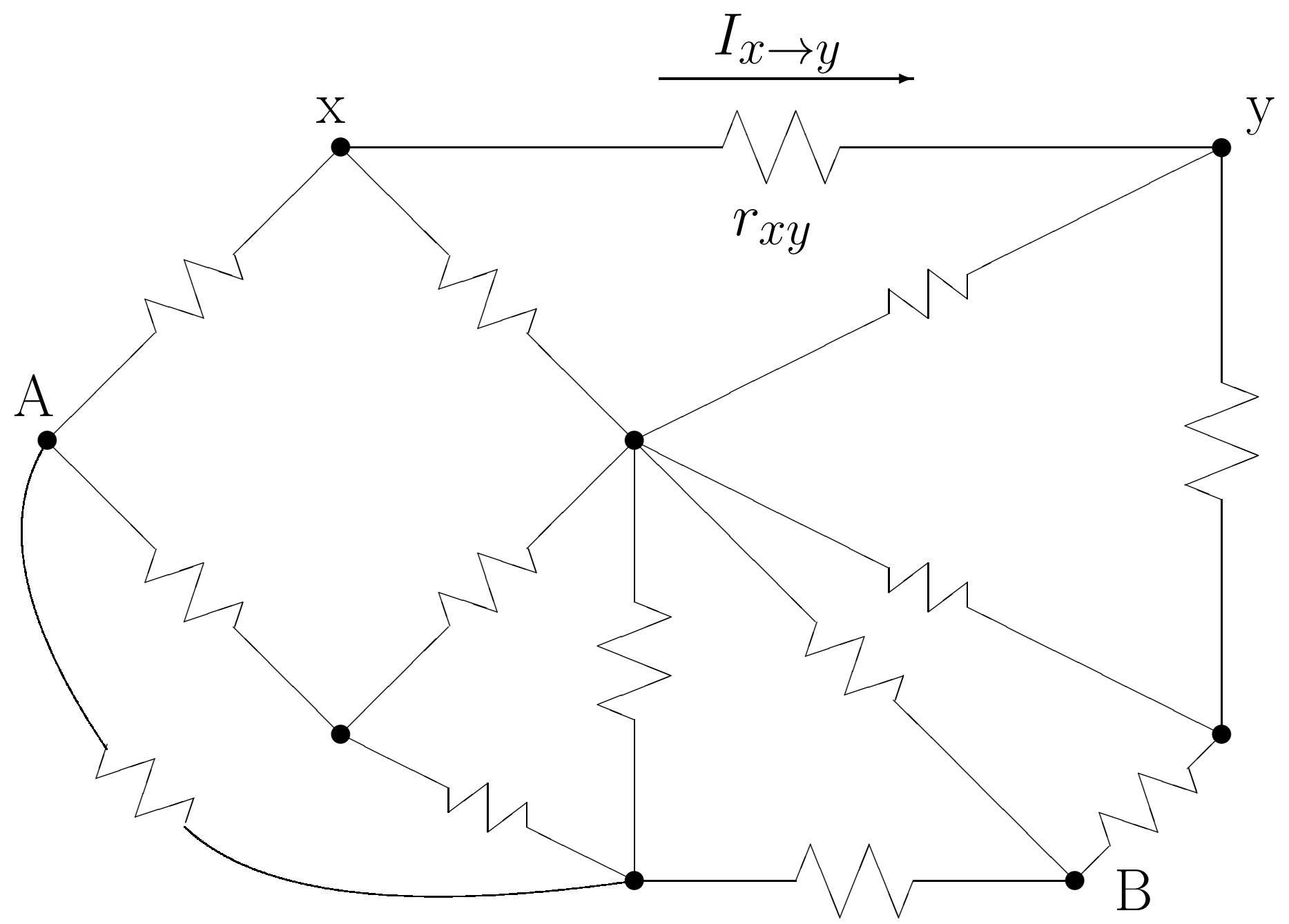

Figure 1

Monwhea Jeng

Random Walks and Effective Resistances on Toroidal and Cylindrical Grids 


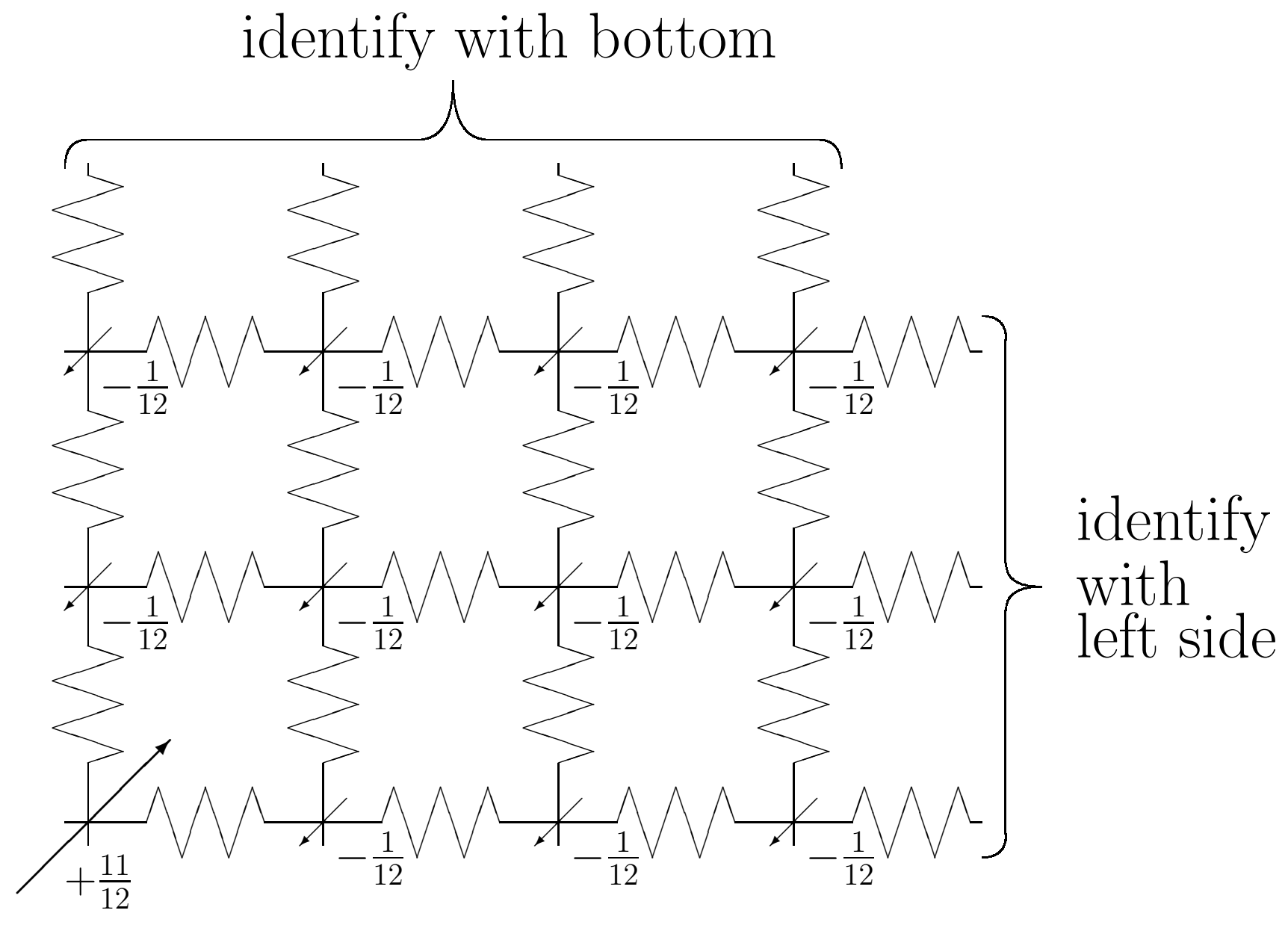

Figure 2

Monwhea Jeng

Random Walks and Effective Resistances on Toroidal and Cylindrical Grids 